\title{
Atomic-Scale Variations of the Mechanical Response of 2D Materials Detected by Noncontact Atomic Force Microscopy
}

\author{
B. de la Torre, ${ }^{1}$ M. Ellner, ${ }^{2}$ P. Pou,${ }^{2,3}$ N. Nicoara, ${ }^{1,4}$ Rubén Pérez,${ }^{2,3, *}$ and J. M. Gómez-Rodríguez ${ }^{1,3,5, \dagger}$ \\ ${ }^{1}$ Departamento de Fisica de la Materia Condensada, Universidad Autónoma de Madrid, E-28049 Madrid, Spain \\ ${ }^{2}$ Departamento de Fisica Teorica de la Materia Condensada, Universidad Autónoma de Madrid, E-28049 Madrid, Spain \\ ${ }^{3}$ Condensed Matter Physics Center (IFIMAC), Universidad Autónoma de Madrid, E-28049 Madrid, Spain \\ ${ }^{4}$ International Iberian Nanotechnology Laboratory, 4715-310 Braga, Portugal \\ ${ }^{5}$ Instituto Nicolás Cabrera, Universidad Autónoma de Madrid, E-28049 Madrid, Spain
}

(Received 5 October 2015; revised manuscript received 1 April 2016; published 16 June 2016)

\begin{abstract}
We show that noncontact atomic force microscopy (AFM) is sensitive to the local stiffness in the atomicscale limit on weakly coupled 2D materials, as graphene on metals. Our large amplitude AFM topography and dissipation images under ultrahigh vacuum and low temperature resolve the atomic and moiré patterns in graphene on $\mathrm{Pt}(111)$, despite its extremely low geometric corrugation. The imaging mechanisms are identified with a multiscale model based on density-functional theory calculations, where the energy cost of global and local deformations of graphene competes with short-range chemical and long-range van der Waals interactions. Atomic contrast is related with short-range tip-sample interactions, while the dissipation can be understood in terms of global deformations in the weakly coupled graphene layer. Remarkably, the observed moiré modulation is linked with the subtle variations of the local interplanar graphene-substrate interaction, opening a new route to explore the local mechanical properties of 2D materials at the atomic scale.
\end{abstract}

DOI: 10.1103/PhysRevLett.116.245502

Atomic force microscopy (AFM) and scanning tunneling microscopy (STM) are tools of choice for characterizing the unique mechanical and electronic properties of graphene $(\mathrm{G})$ and other 2D materials. Dynamic AFM [1] in the frequency modulation (FM) mode [2] has resolved the true geometric structure of a broad range of materials [3-5]. FM AFM experiments on carbon-based materials [6-13] show atomic contrast in $\Delta f$ images and, depending on the setup, in the dissipation channel. While the origin of the dissipation is not well understood, the $\Delta f$ contrast has been linked with the nature of the tip-sample interaction [14].

$\mathrm{G}$ properties can be efficiently tuned by the interaction with metals $[15,16]$. The interaction strength varies widely from the strong coupling with $\mathrm{Rh}[17,18]$ and $\mathrm{Ru}[19]$ to the weak limit (Ir [20], Pt [21]), where G retains its unique electronic properties [22]. The different lattice parameters of $\mathrm{G}$ and the metal underneath are accommodated through the formation of commensurate structures known as moiré patterns, where $\mathrm{C}$ atoms become inequivalent due to their different bonding configuration with the metal. The resulting "true" topographic corrugation of $\mathrm{G}$ - the difference in height among the topmost and the bottom $\mathrm{C}$ atom-varies widely, even in the weakly interacting cases, where it ranges from $\approx 50 \mathrm{pm}$ on Ir $[23,24]$ to practically flat $(\leq 3 \mathrm{pm})$ on $\mathrm{Pt}[21]$.

While STM can easily resolve these moiré patterns, even in the G/Pt case [21,25], AFM experiments have only been reported in highly corrugated cases as $\mathrm{Ru}$ [26], $\mathrm{Rh}$ [27], and Ir $[12,28]$. Focusing on the most challenging case, G/Ir, experiments with a Kolibri sensor using a W tip clearly resolved the moiré in constant height $(\mathrm{CH})$ AFM images [28]. Measurements with a tuning fork using both inert (CO-terminated) and reactive (Ir-terminated) tips [12] were able to identify the atoms with both tips at any tip-sample distance. This atomic-scale resolution allowed the observation of an inversion from attractive to repulsive atomic contrast with decreasing tip-sample distance predicted theoretically for reactive tips [14]. Except for graphite $[9,10]$, atomic resolution in weakly coupled G-based materials using cantilever AFM with large oscillation amplitudes has not been reported.

The origin of the moiré contrast in $\mathrm{G} / \mathrm{Ir}$ was explored with experimental $\Delta f$ vs distance curves on the atop (highest) and $f c c$ (lowest) areas [28]. These curves have minima that differ by $\sim 20 \%$ and are displaced by $\sim 1 \AA$. Density-functional theory (DFT) calculations with a fiveatom $\mathrm{W}$ tip that do not include any atomic relaxations provide very similar interaction energy curves that are only shifted by $0.40 \AA$. Based on this comparison, the CH AFM contrast was attributed to differences in height and interaction strength (not captured by the simulations) for those areas [28].

According to the previous analysis, the quasiflat $\mathrm{G} / \mathrm{Pt}(111)$ poses an insurmountable challenge for AFM imaging. In the absence of a large topographic corrugation, we have to resort to subtle differences in the electronic and mechanical properties of the individual $\mathrm{C}$ atoms induced by the different interaction with the metal substrate. STM on 
$\mathrm{G} / \mathrm{Pt}(111)$ has resolved several moiré patterns [25], whose large apparent corrugation arises from a purely electronic effect [21]. On the mechanical side, AFM has been extensively used to explore the local mechanical properties of materials $[29,30]$ but with lateral resolutions up to the nanometer scale [26,31].

Here we show that large amplitude FM AFM is not only able to achieve atomic resolution on both the $\Delta f$ and dissipation channels but also to resolve the moiré in $\mathrm{G} / \mathrm{Pt}(111)$. The identification of the contrast mechanisms requires the development of a multiscale model based on DFT calculations that takes into account both the global and local deformations in $\mathrm{G}$ induced by the short-range chemical and long-range van der Waals (vdW) interactions. Atomic contrast is related with short-range tip-sample interactions, while dissipation is linked to global deformations of the $\mathrm{G}$ layer. Atom-by-atom stiffness differences due to variations in the coupling with the metal are responsible for the moiré pattern observed both in the attractive and repulsive regimes, showing that AFM is able to map the mechanical properties of $2 \mathrm{D}$ materials at the atomic scale.

Figure 1 shows typical images measured on $\mathrm{G} / \mathrm{Pt}(111)$ with a homemade ultra-high-vacuum NC AFM operated at 5 K with PtIr-coated Si tip cantilevers using large oscillation amplitudes. They are measured at two distinct $\Delta f$ on the same region by using the second-pass method [32]. Both images display not only atomic resolution but also a periodic modulation corresponding to a $3 \times 3$ moiré. The

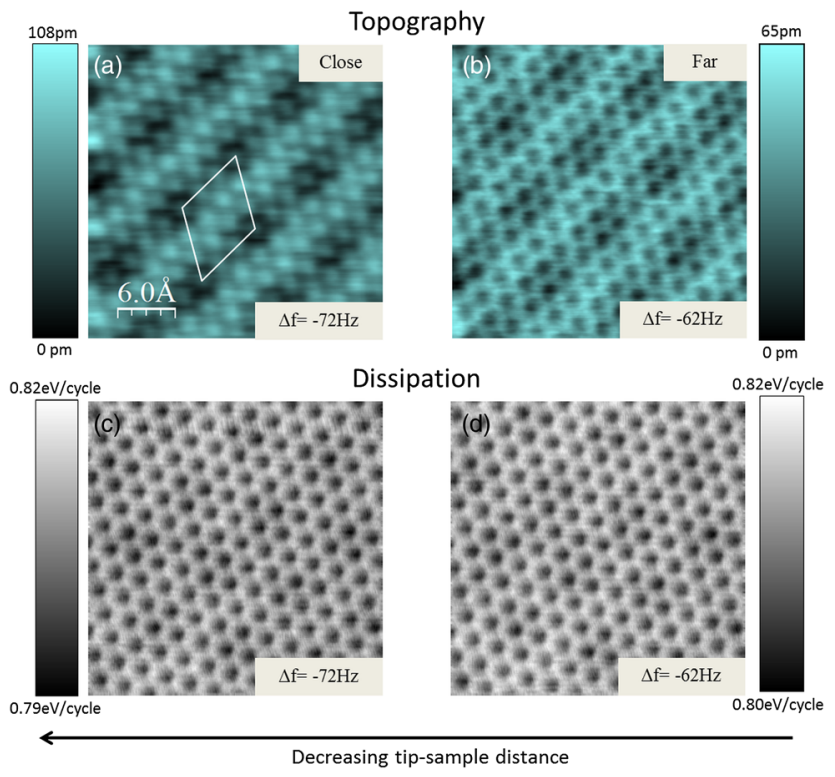

FIG. $1 . \quad 3 \times 3 \mathrm{~nm}^{2} \mathrm{NC}$ AFM simultaneously acquired images on $\mathrm{G} / \mathrm{Pt}(111)$. (a),(b) show topography images at $\Delta f=-72 \mathrm{~Hz}$ and $\Delta f=-62 \mathrm{~Hz}$. The $3 \times 3$ moiré pattern is marked in (a). The corresponding dissipation images are shown in (c) and (d). Parameters: $A=20 \mathrm{~nm} ; V_{\text {bias }}=0 \mathrm{~V}$. All data were acquired with WSXM [38]. atomic-scale features have a honeycomb appearance at low $\Delta f$ [Fig. 1(b)] or a simple triangular lattice, with bright protrusions corresponding to the center of the hexagons, at high $\Delta f$ [Fig. 1(a)]. The low (high) $\Delta f$ corresponds to a cantilever oscillating far (close) from the surface. This atomic contrast behavior on $\mathrm{G}$ could be expected for metallic reactive tips [14]. Strikingly, in both cases, the images show the $3 \times 3$ moire induced by the $\mathrm{Pt}(111)$ substrate. We have measured the dissipation signal [35] simultaneously to the topography [Figs. 1(c) and 1(d)]. Physically, dissipation indicates a hysteresis in the approach and retraction tip path due to nonconservative tip-sample interactions [36,37]. Although both dissipation images show atomic-scale features, no changes upon tip approach are observed.

We have fully characterized the tip-sample interaction by 2D mapping measurements (Fig. 2). In the 2D mapping, the $\Delta f$ [Fig. 2(b)] and dissipation signal [Fig. 2(c)] along a selected line [dashed yellow in Fig. 2(a)] are measured at different tip-sample distances. We have plotted the $\Delta f$ and dissipation vs distance on a topography maximum (red plot) and on a minimum (blue plot). For large tip-sample distances $(Z>0.55 \mathrm{~nm})$, there is no apparent difference between the red and blue plots either in the $\Delta f$ curves (no contrast in topographic images) or in the dissipation. Getting closer to the surface, at $Z=0.55 \mathrm{~nm}$, the

(a)

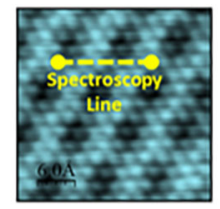

(b)

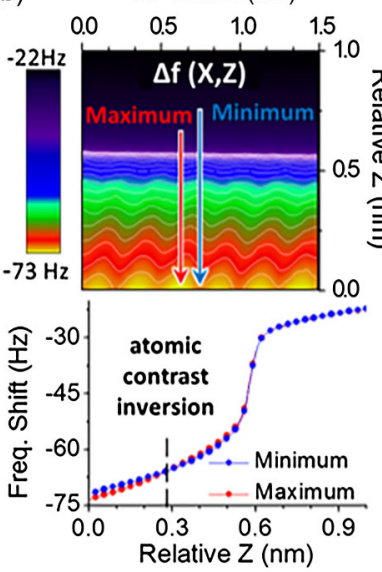

(c) $\quad \times$ Position $(\mathrm{nm})$

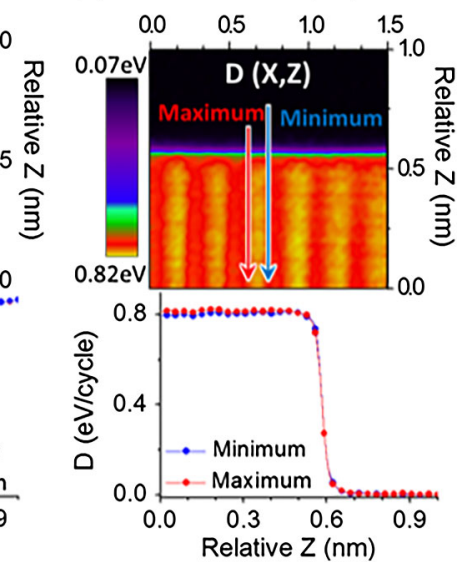

FIG. 2. (a) $3 \times 3 \mathrm{~nm}^{2}$ topographic image acquired at $\Delta f=-73 \mathrm{~Hz}, A=20 \mathrm{~nm}, V_{\text {bias }}=0 \mathrm{~V}$. Two-dimensional plots of (b) $\Delta f$ and (c) energy dissipation measured along the mapping line shown in (a) while varying the tip-sample distance are displayed. Maximum (red) and minimum (blue) in the 2D mapping correspond to maximum and minimum in the topographic image. 
dissipation energy increases abruptly up to a plateau $\approx 0.8 \mathrm{eV} /$ cycle. At this point, atomic features with honeycomb symmetry can be distinguished both in the $\Delta f$ and dissipation images. As the tip-sample distance decreases, the relative corrugation of the dissipation between the hollow and top position stabilizes at less than $0.05 \mathrm{eV} /$ cycle. In contrast, $\Delta f$ slowly inverts its trend. When $Z=0.20 \mathrm{~nm}$, $\Delta f$ over the hollow position becomes more negative resulting in atomic features with triangular symmetry. Dissipation measurements were reproduced using several cantilevers and tips in order to assess the negligible influence of instrumental artifacts due to transfer function effects [39]. More details can be found in Ref. [32].

We have combined DFT calculations with a simple multiscale model to rationalize the experimental findings. We have performed the DFT simulations using the OPENMX code [40] with a double-zeta polarized basis set and the Perdew-Burke-Emzerhof functional [41] supplemented by vdW interactions described using the density
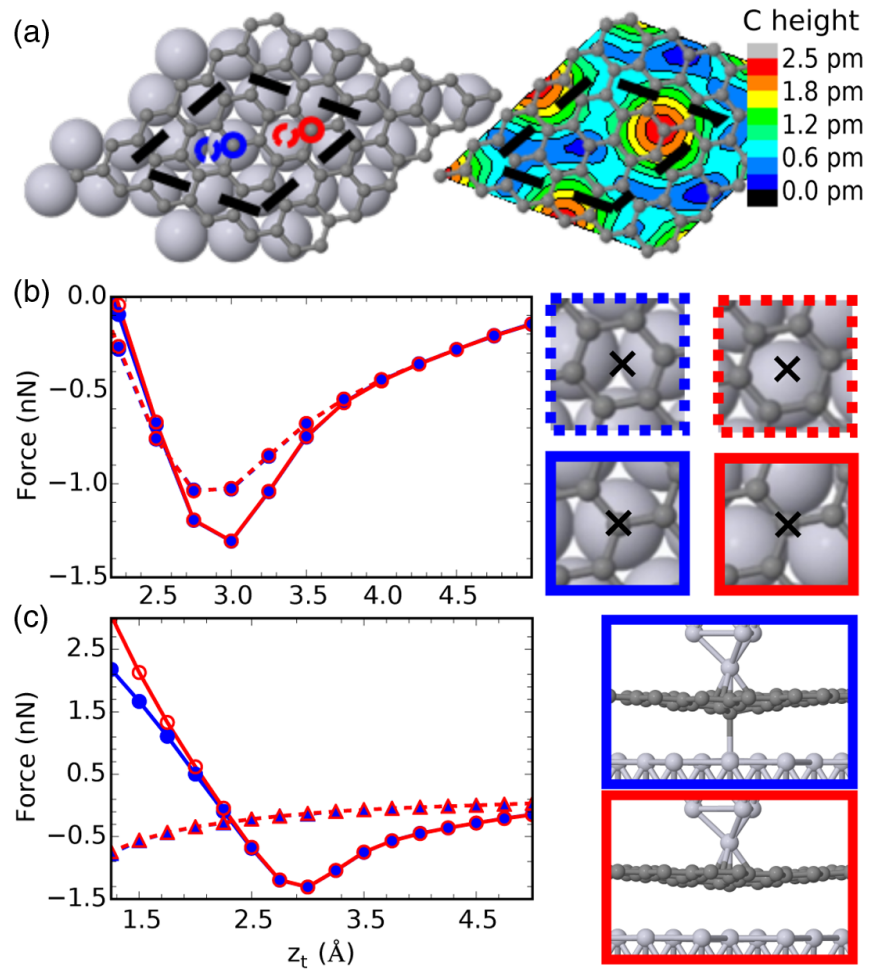

FIG. 3. (a) DFT relaxed G/Pt(111) structure highlighting the low and high moiré areas and the small corrugation. (b) Force vs distance curves in the attractive regime for the four sites marked in (a): top low (solid blue), top high (solid red), hollow low (dashed blue), and hollow high (dashed red). The plot shows atomic contrast on $\mathrm{G}$ and contrast inversion. (c) Different repulsive forces between the top-low and top-high sites for $z_{t}<2.25 \AA$ explain the moiré contrast. Calculations on the bare $\mathrm{Pt}(111)$ surface for these sites (dashed lines and triangles) do not show any force difference thus signaling that the contrast is due to the sensing of the Pt substrate through the displaced $\mathrm{C}$ atoms [insets in panel (c)]. functional dispersion correction approach (DFT-D3) [42]. Figure 3(a) shows the relaxed $6 \times 6$ super cell $(14.85$, 14.85 , and $30 \AA$ ) used in the calculations with a color scale that indicates the relative heights of the $\mathrm{C}$ atoms. The $\mathrm{G}$ layer adheres at $3.35 \AA$ on top of the Pt substrate and remains very flat: the difference in height between the highest $\mathrm{C}$ atom and the lowest is $\approx 2.5 \mathrm{pm}$.

Force versus tip-G distance $\left(z_{t}\right)$ [43] curves calculated with a ten-atom Pt tip and including the tip and sample relaxations (see Ref. [32] for details) are shown in Fig. 3(b). Forces on top of $\mathrm{C}$ atoms (top sites) and on the center of $\mathrm{G}$ hexagons (hollow sites) are indistinguishable for $z_{t}>3.75 \AA$. Below this distance, atomic contrast is obtained and two contrast regimes can be appreciated. First, the forces on the top sites are more attractive than on hollow sites, and at $2.6 \AA$ a change of contrast results in hollow sites being more attractive. Contrast on the moiré superstructure should yield differences on the forces between the top sites. However, for $z_{t}>2.25 \AA$, the force versus distance curves of all top sites are indistinguishable [Fig. 3(b)]. Moreover, dissipation cannot be explained, as no significant difference in the force profile is obtained when comparing the approach and retraction curves.

Although in the attractive regime, DFT calculations do not exhibit moire contrast, this changes for $z_{t} \leq 2.25 \AA$ : the atomic contrast is dominated by the Pauli repulsion and the interaction becomes repulsive [Fig. 3(c)]. In this regime, the contrast between a top site in the low and high areas of the moiré can be appreciated. When removing the $\mathrm{G}$ layer and repeating the calculations only with the Pt substrate, the difference between different sites of the moiré disappears. This shows that the moiré contrast is not due to the direct tip-Pt substrate interaction but to the effect that the substrate has on $\mathrm{G}$ when indenting the tip. This view is supported by calculations in which the $\mathrm{C}$ atoms are kept fixed: these yield no contrast between force curves of different top (or hollow) sites of the moiré. When examining the ionic relaxation [32], it is apparent that in this regime, upon tip approach, the distance between the outermost apex atom of the tip and the $\mathrm{G}$ is only slightly varying. However, the local G-Pt substrate distance changes. The deformation of the $\mathrm{G}$ induced by the tip is so large that the displaced $\mathrm{C}$ atoms are able to sense the Pt substrate: a Pt top site on the topographic low area of the moiré and a Pt hollow on the high area. This interaction changes the local stiffness of the $\mathrm{C}$ atoms that now depends on its location with respect to the Pt substrate. These differences are sensed by the tip while in the repulsive regime and yield the moire pattern contrast. Consequently, this shows the possibility of obtaining atomic subsurface resolution on $G$ on weakly coupled substrates with the AFM.

Our DFT calculations explain the atomic contrast, its inversion, and the moiré contrast in the repulsive regime. However, they fail to reproduce the moire contrast in the attractive regime and the dissipation in the experiments. It 
has been proposed that in STM or AFM experiments on layered materials as graphite, the tips could detach large areas of the last layer from the underneath substrate $[9,44,45]$. This tip-induced detaching could explain our dissipation signal: during tip approach, $\mathrm{G}$ is attached to the substrate, but, upon tip retraction, $G$ locally adheres to the tip temporally, inducing a large-scale deformation of the sheet. This cannot be described with the limited size of DFT simulation cells which prevent us to include the effect of (a) a large $G$ area and (b) a macroscopic tip. The large attractive vdW background induced by a macroscopic tip adds to the short-range tip-G binding and promotes global deformations on an area larger than a few nanometers (we use global for areas larger than the nanometer scale in contrast with the local atomic-scale effective area used in the DFT simulations).

In order to address these shortcomings, we have created a multiscale model characterized through DFT calculations that simulates the tip approach-retraction route taking into account both global and local scales. It includes effective potentials for the interaction of $\mathrm{G}$ with the nanoscopic tip cluster, the macroscopic tip, and the Pt substrate, as well as the elastic response of $\mathrm{G}$ due to out-of-plane deformations [see Fig. 4(a)]. The potential has the form

$$
\begin{aligned}
V\left(z_{\text {global }}, z_{\text {local }}, z_{t}\right)= & V_{t-\mathrm{G}}\left(z_{t}-z_{\text {local }}\right)+A_{1} V_{T-\mathrm{G}}\left(z_{T}-z_{\text {global }}\right) \\
& +A_{1} V_{\mathrm{Pt}-\mathrm{G}}^{\text {global }}\left(z_{\text {global }}-z_{\mathrm{Pt}}\right) \\
& +V_{\mathrm{Pt}-\mathrm{G}}^{\text {local }}\left(z_{\text {local }}\right)+V_{\mathrm{BR}}\left(z_{\text {local }}-z_{\text {global }}\right) \\
& +A_{2} V_{E 2 \mathrm{D}}\left(z_{\text {global }}\right)
\end{aligned}
$$

where $z_{\text {local }}$ is the $z$ coordinate of the $\mathrm{G}$ area just under the tip, and $z_{\text {global }}$ is the mean $z$ coordinate of the globally deformed $\mathrm{G}$ flake, then $z_{\text {local }}-z_{\text {global }}$ measures the local deformation. As before, $z_{t}$ is the nanoscopic tip-G distance. $z_{T}$ is the macroscopic tip-G separation (with $z_{t}+$ $z_{T}=$ const), and $z_{\mathrm{Pt}}$ the position of the Pt surface. All distances are measured with respect to G's equilibrium position without the tip [see Fig. 4(a)]. $V_{t-\mathrm{G}}$ describes the interaction of the nanoscopic apex with $\mathrm{G}$ and includes both $\mathrm{vdW}$ and short-range chemical interactions. $V_{T-\mathrm{G}}$ and
$V_{\mathrm{Pt}-\mathrm{G}}^{\text {global }}$ approximate the mean interaction between the deformed global area of the $\mathrm{G}$ sheet and the macroscopic part of the tip and the $\mathrm{Pt}$ substrate, respectively; they include both dispersion and chemical interactions. $V_{\mathrm{Pt}-\mathrm{G}}^{\text {local }}$ effectively takes into account the interaction of the local area of $\mathrm{G}$ with the Pt substrate; i.e., it incorporates in the model the atomic-scale variations of the G-Pt interaction. $V_{\mathrm{BR}}$ and $V_{E 2 \mathrm{D}}$ describe the energetic response of G to outof-plane deformations [46]. Finally, we note that $V_{T-\mathrm{G}}$, $V_{\mathrm{Pt}-\mathrm{G}}^{\text {global }}$, and $V_{E 2 \mathrm{D}}$ interactions depend on the spatial area of the global deformation. We consider this effect through parameters $A_{1}$ and $A_{2}$ chosen to reproduce the dissipation energy plateau measured in the experiments.

Figure 4(b) shows the full potential of the model with respect to the global and local deformations of $\mathrm{G}$ and for different $z_{t}$. When the tip is far, the system has only one minimum corresponding to $\mathrm{G}$ at its equilibrium position without the tip. As the tip approaches, both the local and global deformations slightly increase. At $z_{t} \approx 5.5 \AA$, a new local minimum appears at $z_{\text {global }} \approx 2.5 \AA$ away from the G's equilibrium position. As the distance is further reduced, the two minima start to converge towards each other. At $z_{t} \approx 3.5 \AA$, the second minimum becomes the global minimum but quickly merges with the first one. The potential profile is summarized in Fig. 4(c) where the energy vs the reaction coordinate is plotted.

The approach-retract procedure presents two types of cycles depending on whether the retraction occurs before or after the energy barrier between the two energy minima disappears at $z_{t}=3.5 \AA$. In both cases, as the tip approaches, the system follows the first solution [left minimum in Fig. 4(c)]. If the tip retracts before the barrier, it returns through the first minimum. However, if it retracts after the barrier, the tip and $\mathrm{G}$ adhere, and the system jumps to the second minimum [right minimum in Fig. 4(c)] and retracts through this minimum until $z_{t}=5.5 \AA$, where $\mathrm{G}$ disadheres from the tip, and the system jumps back to the first minimum. The difference in energy between the two solutions is $0.8 \mathrm{eV}$ which corresponds to the dissipation plateau, i.e., $0.8 \mathrm{eV} /$ cycle, observed in the experiments.
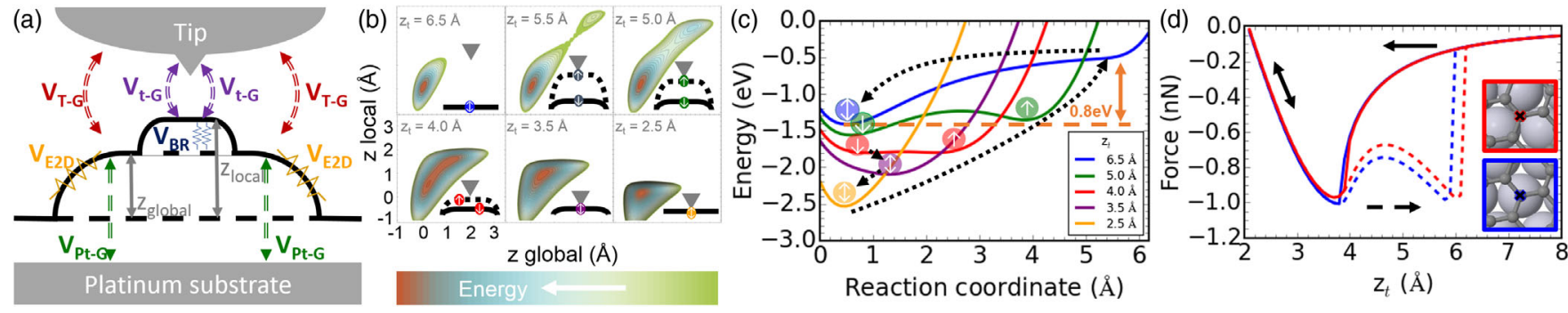

FIG. 4. (a) A scheme of the theoretical model which includes a macroscopic and nanoscopic Pt tip, the G surface divided into a local and global area, and the Pt substrate. For different tip heights, $z_{t}$, (b) 2D minimization of the potential with respect to the local and global deformation and (c) energy profile versus the reaction coordinate. Heights are referenced to the equilibrium position of the $\mathrm{G}$ on Pt. (d) Calculated force vs distance curves for the tip approach (solid) and retraction (dashed) in moiré top-high (red) and top-low (blue) sites. 
In order to further compare with experiments, Fig. 4(d) shows force vs distance curves simulated with the multiscale model. Upon indentation, the shapes of the curves follow those obtained from DFT (Fig. 3). However, upon retraction, $\mathrm{G}$ adheres to the tip, resulting in a large attractive force, keeping the separation between the tip apex and the closest atoms of $\mathrm{G}$ near constant, while these atoms separate from the Pt substrate. Furthermore, the model confirms contrast on the moiré: force curves for the tip at high and low moiré positions are different. This comes from the $V_{\mathrm{Pt}-\mathrm{G}}^{\text {local }}$ term that captures the small local variations of Pt-G interaction. The AFM can sense them at the attractive regime due to the large $G$ deformation induced by the tip during the retraction. This result confirms that the sensing of the moire in the attractive regime is mapping the local stiffness of $\mathrm{G}$.

In summary, our NC AFM measurements not only provide atomic contrast, but they are able to resolve the moiré pattern formed on the very flat $\mathrm{G} / \mathrm{Pt}(111)$. A combination of DFT simulations and a multiscale model designed to capture the subtle interplay between local and global tip-induced deformations unveils the imaging mechanisms. Dissipation is due to the adhesion of $G$ to the apex upon tip retraction which induces deformations on largescale areas of G. This tip-induced deformation appears in STM and AFM experiments on layered materials. The contrast in the moiré pattern originates from the small local differences on the metal-G interaction. They can be measured by the AFM because the tip displaces the atoms of the $\mathrm{G}$ layer on both the attractive and repulsive regimes. Therefore, our results show that the AFM experiments on 2D materials can unveil not only the topography of the sample and its interaction with the tip probe but also the interaction of the layer with the underneath substrate at the atomic scale.

We thank the Marie Curie ITN Network "ACRITAS" (Grant No. FP7-PEOPLE-2012-ITN-317348) funded by the European Commission under the FP7 Marie Curie PEOPLE programme and the Spanish MINECO (Projects No. CSD2010-00024, No. MAT2011-23627, No. MAT2013-41636-P, and No. MAT2014-54484-P) for financial support. Computer time was provided by the Spanish Supercomputer Network (RES) at Marenostrum III (BSC, Barcelona) and Magerit (CesViMa, Madrid) computers. P. P. was supported by the Ramón y Cajal program.

B. de la T. and M. E. contributed equally to this work.

*ruben.perez@uam.es †josem.gomez@uam.es

[1] R. Garcia and R. Perez, Surf. Sci. Rep. 47, 197 (2002).

[2] Noncontact Atomic Force Microscopy, edited by S. Morita, F. J. Giessibl, and R. Wiesendanger (Springer, Berlin, 2009), Vol. 2.
[3] L. Gross, F. Mohn, N. Moll, P. Liljeroth, and G. Meyer, Science 325, 1110 (2009).

[4] Y. Sugimoto, P. Pou, M. Abe, P. Jelinek, R. Perez, S. Morita, and O. Custance, Nature (London) 446, 64 (2007).

[5] L. Gross, F. Mohn, N. Moll, B. Schuler, A. Criado, E. Guitian, D. Pena, A. Gourdon, and G. Meyer, Science 337, 1326 (2012).

[6] W. Allers, A. Schwarz, U.D. Schwarz, and R. Wiesendanger, Appl. Surf. Sci. 140, 247 (1999).

[7] H. Holscher, A. Schwarz, W. Allers, U. D. Schwarz, and R. Wiesendanger, Phys. Rev. B 61, 12678 (2000).

[8] S. Hembacher, F. J. Giessibl, J. Mannhart, and C. F. Quate, Proc. Natl. Acad. Sci. U.S.A. 100, 12539 (2003).

[9] S. Kawai and H. Kawakatsu, Phys. Rev. B 79, 115440 (2009).

[10] S. Kawai, T. Glatzel, S. Koch, B. Such, A. Baratoff, and E. Meyer, Phys. Rev. B 81, 085420 (2010).

[11] M. Ashino, A. Schwarz, T. Behnke, and R. Wiesendanger, Phys. Rev. Lett. 93, 136101 (2004).

[12] M. P. Boneschanscher, J. van der Lit, Z. X. Sun, I. Swart, P. Liljeroth, and D. Vanmaekelbergh, ACS Nano 6, 10216 (2012).

[13] J. van der Lit, M. P. Boneschanscher, D. Vanmaekelbergh, M. Ijas, A. Uppstu, M. Ervasti, A. Harju, P. Liljeroth, and I. Swart, Nat. Commun. 4, 2023 (2013).

[14] M. Ondracek, P. Pou, V. Rozsival, C. Gonzalez, P. Jelinek, and R. Perez, Phys. Rev. Lett. 106, 176101 (2011).

[15] J. Wintterlin and M. L. Bocquet, Surf. Sci. 603, 1841 (2009).

[16] S. Bae, H. Kim, Y. Lee, X. F. Xu, J. S. Park, Y. Zheng, J. Balakrishnan, T. Lei, H. R. Kim, Y. I. Song, Y. J. Kim, K. S. Kim, B. Ozyilmaz, J. H. Ahn, B. H. Hong, and S. Iijima, Nat. Nanotechnol. 5, 574 (2010).

[17] B. Wang, M. Caffio, C. Bromley, H. Fruchtl, and R. Schaub, ACS Nano 4, 5773 (2010).

[18] A. Martin-Recio, C. Romero-Muñiz, A. J. Martinez-Galera, P. Pou, R. Perez, and J. M. Gomez-Rodriguez, Nanoscale 7, 11300 (2015).

[19] B. Borca, S. Barja, M. Garnica, M. Minniti, A. Politano, J. M. Rodriguez-Garcia, J. J. Hinarejos, D. Farias, A. L. V. de Parga, and R. Miranda, New J. Phys. 12, 093018 (2010).

[20] I. Pletikosic, M. Kralj, P. Pervan, R. Brako, J. Coraux, A. T. N'Diaye, C. Busse, and T. Michely, Phys. Rev. Lett. 102, 056808 (2009).

[21] M. M. Ugeda, D. Fernandez-Torre, I. Brihuega, P. Pou, A. J. Martinez-Galera, R. Perez, and J. M. Gomez-Rodriguez, Phys. Rev. Lett. 107, 116803 (2011).

[22] A. H. C. Neto, F. Guinea, N. M. R. Peres, K. S. Novoselov, and A. K. Geim, Rev. Mod. Phys. 81, 109 (2009).

[23] S. K. Hamalainen, M. P. Boneschanscher, P. H. Jacobse, I. Swart, K. Pussi, W. Moritz, J. Lahtinen, P. Liljeroth, and J. Sainio, Phys. Rev. B 88, 201406(R) (2013).

[24] F. Jean, T. Zhou, N. Blanc, R. Felici, J. Coraux, and G. Renaud, Phys. Rev. B 91, 245424 (2015).

[25] P. Merino, M. Svec, A. L. Pinardi, G. Otero, and J. A. Martin-Gago, ACS Nano 5, 5627 (2011).

[26] S. Koch, D. Stradi, E. Gnecco, S. Barja, S. Kawai, C. Diaz, M. Alcami, F. Martin, A. L. V. de Parga, R. Miranda, T. Glatzel, and E. Meyer, ACS Nano 7, 2927 (2013). 
[27] E. N. Voloshina, Y. S. Dedkov, S. Torbrugge, A. Thissen, and M. Fonin, Appl. Phys. Lett. 100, 241606 (2012).

[28] E. N. Voloshina, E. Fertitta, A. Garhofer, F. Mittendorfer, M. Fonin, A. Thissen, and Yu. S. Dedkov, Sci. Rep. 3, 1072 (2013).

[29] U. Landman, W. D. Luedtke, N. A. Burnham, and R. J. Colton, Science 248, 454 (1990).

[30] R. Garcia and E. T. Herruzo, Nat. Nanotechnol. 7, 217 (2012).

[31] T. Herden, M. Ternes, and K. Kern, Nano Lett. 14, 3623 (2014).

[32] See the Supplemental Material at http://link.aps.org/ supplemental/10.1103/PhysRevLett.116.245502 for more details on the determination of the multiscale model and the experimental methods, which includes Refs. [33,34] and two videos extracted from DFT simulations.

[33] J. P. Cleveland, S. Manne, D. Bocek, and P. K. Hansma, Rev. Sci. Instrum. 64, 403 (1993).

[34] A. J. Martínez-Galera and J. M. Gómez-Rodríguez, J. Phys. Chem. C 115, 23036 (2011).

[35] C. Loppacher, R. Bennewitz, O. Pfeiffer, M. Guggisberg, M. Bammerlin, S. Schar, V. Barwich, A. Baratoff, and E. Meyer, Phys. Rev. B 62, 13674 (2000).

[36] N. Oyabu, P. Pou, Y. Sugimoto, P. Jelinek, M. Abe, S. Morita, R. Perez, and O. Custance, Phys. Rev. Lett. 96, 106101 (2006).
[37] L. N. Kantorovich and T. Trevethan, Phys. Rev. Lett. 93, 236102 (2004)

[38] I. Horcas, R. Fernandez, J. M. Gomez-Rodriguez, J. Colchero, J. Gomez-Herrero, and A. M. Baro, Rev. Sci. Instrum. 78, 013705 (2007).

[39] A. Labuda, Y. Miyahara, L. Cockins, and P. H. Grutter, Phys. Rev. B 84, 125433 (2011).

[40] T. Ozaki, Phys. Rev. B 67, 155108 (2003).

[41] J. P. Perdew, K. Burke, and M. Ernzerhof, Phys. Rev. Lett. 77, 3865 (1996).

[42] S. Grimme, J. Antony, S. Ehrlich, and H. Krieg, J. Chem. Phys. 132, 154104 (2010).

[43] $z_{t}$ is defined as the distance between the fixed layers in the tip and sample, so it is directly comparable to the piezo displacement measured in the experiments. An appropriate constant has been subtracted, so it corresponds to the distance between the tip apex atom and the $\mathrm{G}$ layer at large distances where no relaxations take place.

[44] J. M. Soler, A. M. Baró, N. García, and H. Rohrer, Phys. Rev. Lett. 57, 444 (1986).

[45] S. Hembacher, F. J. Giessibl, J. Mannhart, and C. F. Quate, Phys. Rev. Lett. 94, 056101 (2005).

[46] C. Lee, X. D. Wei, J. W. Kysar, and J. Hone, Science 321, 385 (2008). 